\title{
Spectrum of Biopsy Proven Extraocular Muscle Tumours of Non-Thyroid Origin
}

\author{
Tayyab Afghani1, Hassan Mansoor1, Amna Manzoor Mughal1, Mohammad Asif1, \\ Muhammad Asif2 and Naeem Raza Hamdani2
}

\begin{abstract}
Objective: To describe different types of primary extraocular muscle (EOM) tumours based on the results of imaging studies, peroperative clinical picture and their histopathological diagnosis.

Study Design: Case series.

Place and Duration of Study: Al-Shifa Trust Eye Hospital, Rawalpindi, from July 2001 to January 2017.

Methodology: A retrospective analysis of 640 diagnosed orbital tumours was carried out using non-randomised sampling technique, and the prevalence of primary EOM tumours was determined. Based on the results of imaging studies, the clinical picture observed during surgery (orbitotomy) and the histopathological diagnosis, primary EOM tumours were divided into different types, accordingly.

Results: Nineteen $(n=19)$ primary EOM tumours (frequency of 2.96\%) had 12 types of histopathological diagnoses, and were categorised into inflammatory tumours $(n=8,42 \%)$, vascular tumours $(n=4,21 \%)$, lymphoproliferative tumours $(n=3$, $16 \%)$, neurogenic tumours $(n=2,10.5 \%)$ and myogenic tumours $(n=2,10.5 \%)$. The recti were involved more frequently than obliques ( $n=15,78.94 \%$ and $n=4,21.06 \%$, respectively). All the patients presented with proptosis of varying degree with some degree of globe rotation and had surgical excision/appropriate management. Visual acuity was not affected in any of the patients. Four ( $n=4,21.05 \%)$ tumours were malignant (NHL, ASPS, myeloid sarcoma and rhabdomyosarcoma) and these patients underwent chemotherapy and/or radiotherapy.

Conclusion: Biopsy-proven primary EOM tumours were devisable into five broad categories. Patients with primary EOM tumours presented with proptosis and impaired ocular motiliy. The primary EOM tumours involved both the recti and the obliques and were excised surgically with favourable outcomes in most cases.
\end{abstract}

Key Words: Biopsy, Extraocular muscle, Tumour, Proptosis.

\section{INTRODUCTION}

Cranial mesoderm which surrounds the developing eye gives rise to extraocular muscles. ${ }^{1}$ Each orbit has seven muscles: four recti, superior and inferior obliques and a levator palpebrae superioris. ${ }^{2}$ Thyroid orbitopathy is the most common cause of extraocular muscle abnormalities. However, various vascular, inflammatory, neoplastic, infective, metabolic and neuromuscular disorders could affect the extraocular muscles morphology and their functions. ${ }^{3}$

Primary extraocular muscle (EOM) tumours are rare. ${ }^{3}$ Their exact incidence is difficult to calculate as they could be secondary to a generalised orbital disease, such as infiltrating tumours and non-specific orbital inflammatory syndrome. ${ }^{4}$ The authors believe that this study presents the largest case series on biopsy-proven

1 Department of Orbit and Oculoplastics, Al-Shifa Trust Eye Hospital, Jhelum Road, Rawalpindi, Pakistan

2 Department of Histopathology, Armed Forces Institute of Pathology (AFIP), Rawalpindi, Pakistan

Correspondence: Dr. Hassan Mansoor, Department of Orbit and Oculoplastics, Al-Shifa Trust Eye Hospital, Jhelum Road, Rawalpindi, Pakistan

E-mail: hassan-mansoor@hotmail.com

Received: April 05, 2018; Accepted: January 08, 2019 primary extraocular muscle tumours, reported in the international literature to date. The authors have also reported a case of alveolar soft-part sarcoma, involving the EOM primarily.

\section{METHODOLOGY}

The study was conducted from July 2001 to January 2017. A retrospective analysis of 640 diagnosed orbital tumours was carried out. The frequency of primary extraocular muscle tumours was determined. The clinical features of the included patients and their subsequent management were reported. The sampling technique was non-randomised sampling. The Institutional Ethical and Research Committee approved the study.

All the patients included in the retrospective analysis underwent orbitotomy after the necessary imaging studies and the diagnoses were confirmed on histopathology. The patients with radiological and histopathological evidence of having a primary extraocular muscle tumour were included in the current case series. Based on the results of imaging studies, the peroperative clinical picture, and their histopathological diagnosis, the primary extraocular muscle tumours were categorised into five different categories, accordingly. Patients with an orbital neoplasm, which could not be confirmed as a primary extraocular muscle tumour on imaging studies or histopathology, were excluded from the current case series. 
Attempts were made to preserve the structure and the function of the involved extraocular muscle as much as possible that was followed by subsequent necessary management. Moreover, in some cases, it was seen that the eye was deviated opposite to the direction of the action of the involved muscle with primary EOM tumour, necessitating further intervention to correct the ocular deviation. The function of the muscle with primary EOM tumour gradually returned to normal or near normal with time in some cases.

Frequency of categorical and mean $+S D$ values of quantitative variables was determined.

\section{RESULTS}

Of the 640 orbital neoplasms, there were 19 primary extraocular muscle tumours with frequency of $2.96 \%$. The 19 different $(n=19)$ tumours had 12 types of histopathological diagnoses, which have been divided into five broad categories, following the usual classification system for the orbital tumours, as shown in Table I.

There were $11(n=11 ; 57.8 \%)$ males and eight $(n=8 ; 42.2 \%)$ females. The mean age was of $22.52 \pm 17.06$ years, ranging from 4 to 64 years. All the patients were Pakistanis by nationality and represented different ethnic groups (Punjabi, Sindhi, Baloch and Pakhtoon). The recti were involved in 15 cases $(n=15 ; 78.94 \%)$ as compared to the oblique muscles, which were involved in just four cases $(n=4 ; 21.06 \%)$. The medial and the inferior quadrants were involved more frequently than the other quadrants. Except for one patient with angiolymphoid hyperplasia, where the involvement of the orbital floor was also seen, the rest of the patients had an isolated single-muscle involvement only. In addition, one patient presented with spontaneous intramuscular haemorrhage. Besides, those patients were included in the current case series who presented with proptosis and globe rotation of varying degrees. However, the visual acuity was not affected in any of the patients. In addition, no patient in the current study showed any signs of muscle shrinkage secondary to cranial nerve palsy, congenital fibrosis or postinflammatory fibrosis.

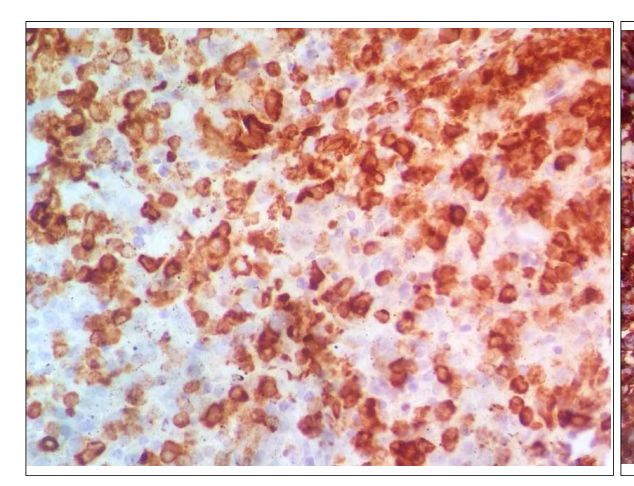

Figure 1: The figure shows positive staining for myeloperoxidase but negative staining for $B$ and $T$

cell markers in a myeloid sarcoma patient.
Inflammatory tumours in the form of fungal granulomas, nonspecific granulomas, and idiopathic orbital inflammatory disease were the commonest and represented $42 \%(n=8)$ of the diagnosed cases. Seemingly, the inflammatory tumours had a male preponderance and were seen in five $(n=5,63 \%)$ male and three $(n=3,37 \%)$ female patients. Besides, all the patients with fungal granuloma underwent systemic antifungal therapy with itraconazole following orbitotomy.

Vascular tumours like cavernous haemangioma and angiolymphoid hyperplasia were the second most common entity, involving $21 \%(n=4)$ of the diagnosed cases with primary EOM tumours. Moreover, vascular tumours were found equally in both male $(n=2,50 \%)$ and female $(n=2,50 \%)$ patients in the current case series.

The lymphoproliferative lesions, like extranodal marginal zone lymphomas, myeloid sarcoma (Figure 1) and the pseudolymphoma (Figure 2) were found in $16 \%(n=3)$ of the included patients. In addition, the lymphoproliferative tumours were more common in females $(n-2,67 \%)$ than males $(n=1,33 \%)$.

Neurogenic and myogenic tumours were found in two patients $(n=2 ; 10.5 \%)$ each. Neurogenic tumours were seen in male patients $(n=2,100 \%)$ only; whereas, myogenic tumours involved male $(n=1,50 \%)$ and female $(n=1,50 \%)$ patients equally.

Four $(21.05 \%)$ of the 19 tumours were malignant in nature (NHL, ASPS, Myeloid Sarcoma and Rhabdomyosarcoma) and underwent appropriate chemotherapy and/or radiotherapy. One child $(n=1 ; 5 \%)$ with rhabdomyosarcoma, died because of the metastatic disease, after two years. One child $(n=1 ; 5 \%)$ with fungal granuloma developed an intracranial lesion, which was found to be of tuberculous in origin, after the neurosurgical intervention. Eleven $(n=11 ; 57.8 \%)$ out of the 19 patients had some degree of loss of muscle function after the treatment.

\section{DISCUSSION}

Lacey et al. reported $31 \quad(n=31)$ biopsy-proven EOM tumours, which included $16(n=16)$ cases of primary

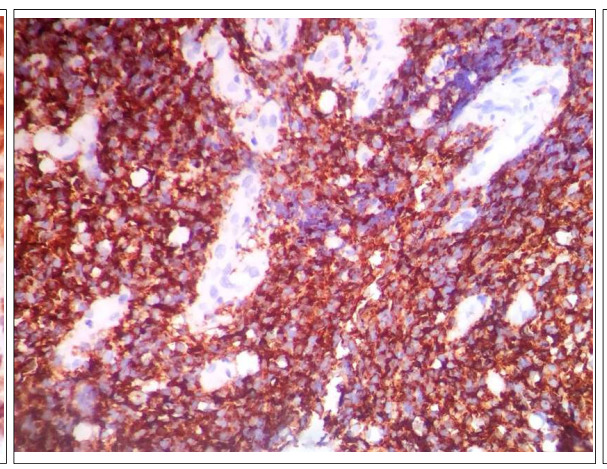

Figure 2: Positive CD 20 staining of $B$ cells in psuedolymphoma patient.

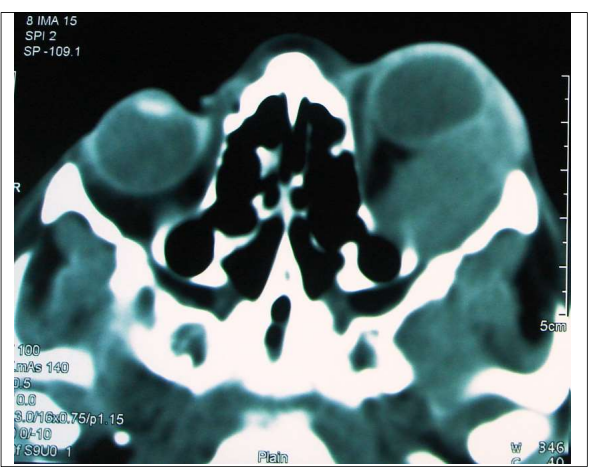

Figure 3: CT scan image showing alveolar soft part sarcoma of left lateral rectus muscle. 
Table I: Outcome after treatment of EOM tumours.

\begin{tabular}{|c|c|c|c|c|c|c|}
\hline SN & Age & Gender & Diagnosis & EOM involved & Treatment & Follow-up and outcome \\
\hline \multicolumn{7}{|c|}{ A. Inflammatory tumours } \\
\hline 1. & 21 years & M & Non-specific chronic granuloma & R-Medial rectus & Partial excision of the muscle tumour & $\begin{array}{l}4 \text { years. No recurrence. Limitation of } \\
\text { medial rectus movements }\end{array}$ \\
\hline 2. & 18 years & $\mathrm{F}$ & Pseudotumour / myositis & R-Medial rectus & $\begin{array}{l}\text { Biopsy of muscle preceded and followed } \\
\text { by steroids }\end{array}$ & Symptom-free after six years \\
\hline 3. & 25 years & $M$ & Fungal granuloma & R-Inferior rectus & $\begin{array}{l}\text { Partial excision of muscle tumour } \\
\text { followed by nine months treatment with } \\
\text { Itraconazole }\end{array}$ & $\begin{array}{l}\text { No recurrence. Inferior rectus limitation } \\
\text { gradually improved over } 3 \text { years with } \\
\text { mild hypertropia persisting }\end{array}$ \\
\hline 4. & 4 years & M & $\begin{array}{l}\text { Fungal granuloma with abscess } \\
\text { formation }\end{array}$ & L-Inferior rectus & $\begin{array}{l}\text { Partial excision of muscle tumour followed } \\
\text { by six months treatment with Itraconazole }\end{array}$ & $\begin{array}{l}\text { Lost to follow-up after } 3 \text { years. The child } \\
\text { had small degree of hypertopia at the } \\
\text { last follow-up. }\end{array}$ \\
\hline 5. & 45 years & M & Fungal granuloma & R-Medial rectus & $\begin{array}{l}\text { Total excision of muscle tumour followed } \\
\text { by nine months treatment with } \\
\text { Itraconazole }\end{array}$ & $\begin{array}{l}\text { No recurrence after two years. Large } \\
\text { exotropia persists. Muscle surgery } \\
\text { planned. }\end{array}$ \\
\hline 6. & 40 years & $\mathrm{F}$ & Non-specific chronic granuloma & $\begin{array}{l}\mathrm{R} \text { - Medial } \\
\text { rectus }\end{array}$ & $\begin{array}{l}\text { Partial excision of muscle tumour } \\
\text { preceded and followed by steroids }\end{array}$ & Symptom-free after one year \\
\hline 7. & 08 years & $\mathrm{F}$ & Inflamatory psuedotumour & $\begin{array}{l}\text { Left superior } \\
\text { oblique }\end{array}$ & $\begin{array}{l}\text { The tumour involving distal portion of } \\
\text { muscle near insertion was excised in toto } \\
\text { preserving the full length of superior oblique. }\end{array}$ & $\begin{array}{l}\text { Tumour-free and symptom free follow } \\
\text { up of eight months }\end{array}$ \\
\hline 8. & 18 years & M & $\begin{array}{l}\text { Right fungal granuloma orbit and } \\
\text { tuberculoma in brain }\end{array}$ & R-Inferior rectus & $\begin{array}{l}\text { Partial excision of muscle tumour } \\
\text { followed by nine months treatment with } \\
\text { Itraconazole. Neurosurgeon removed the } \\
\text { tuberculoma from frontal lobe and } \\
\text { antituberculous therapy was given. }\end{array}$ & $\begin{array}{l}\text { Moderate hypertropia persists after four } \\
\text { years of follow-up. }\end{array}$ \\
\hline
\end{tabular}

\section{B. Vascular tumours}

Cavernous hemangioma

Left-Inferior

oblique

10. 15 years $\mathrm{F}$

Intermuscular hemangioma

11. 08 years F

Angiolymphyoid hyperplasia

12. 38 years $M$

Angiolymphyoid hyperplasia
Medial rectus

Left -lateral

rectus

Left-lateral rectus and floor of the orbit
Tumour involving the middle third of muscle belly was excised while the muscle was preserved

Hemangioma was partially excised and drained

Total excision of the tumour along with the muscle.

Excision biopsy of the lateral rectus and removal of orbital floor tumour.
Symptom-free one year follow-up

Two years follow-up without any symptom

Follow-up of two years. Left large esotropia. Muscle surgery for squint planned

Moderate proptosis with mild esotropia persists after one year

\section{Lymphoproliferative tumours}

13. 45 years $F$

14. 11 years $M$

15. 32 years $F$
Malignant non hodgkin's lymphoma (extranodal marginal zone lymphoma)

Right granulocytic sarcoma

Pseudolymphoma
Left-inferior oblique

Right superior rectus

Right medial rectus
Partial excision of the tumour. Systemic workup was negative. Local radiation was given.

Systemic workup revealed acute lymphoblastic leukemia and child was referred to pediatric oncologist for chemotherapy
Tumour and symptom- free after three years

Lost to follow-up after six months. Child was with large hypotropia and undergoing chemotherapy at the last follow up

\section{Neurogenic tumours}

12 years $M$

17. 2 years $M$

Schwannomma

Glomus tumour of orbit (paraganglioma) with chronic granulomatous tissue-
Left superior Tumour was excised from the muscle oblique

Right superior rectus near trochlea.

Excision of the tumour involving the superior rectus.
Tumour and symptom-free after five years of follow-up

Complete ptosis, limitation of upgaze after one year of follow-up

\section{E. Myogenic tumours}

18. 05 years $M$

Embryonal rhabdomyosarcoma

19. 17 years $F$
Alveolar soft-part sarcoma of orbit
Right-inferior

rectus

Left lateral

rectus
Total excision of the tumour along with the muscle followed by radiation and chemotherapy

Lateral orbitotomy revealed a large tumour of lateral rectus muscle which was partially excised. It was followed by chemotherapy and radiation.
The child had large hypertropia died with recurrence after two years.

Six months follow-up after surgery, radiation and chemotherapy show no recurrence with small degree of esotropia. 
muscle involvement while the rest involved the extraocular muscles secondarily, either due to the distant metastasis $(n=8)$ or the local invasion, from the adjacent orbital neoplasms $(n=7) \cdot{ }^{3}$ The authors in the current study report $19(n=19)$ biopsy-proven primary extraocular muscle tumours, making it the largest case series, reported in the international literature to date (Table I).

The signs and symptoms of patients, with primary extraocular muscle tumours in the current study, were comparable with the findings of Lacey et al. ${ }^{3}$ However, contrary to the conclusions from Lacey et al., ${ }^{3}$ carotidcavernous dural shunts, congenital and acquired fibrosis of the extraocular muscles, systemic associations especially neurofibromatosis, systemic inflammatory/ autoimmune disorders and the amyloid deposition in the extraocular muscles, were not seen by the authors in this case series.

In this study, a male preponderance was noted $(58 \%$, $\mathrm{n}=11$ ), which was also seen in the study conducted by Mannor et al. ${ }^{5}$ Contrary to this, Lacey et al. ${ }^{3}$ and Scott and Siatkowski, ${ }^{6}$ showed a female preponderance. The average age in the current study (mean age 22 years 5 months) was less (4-5th decade) than the one reported in the studies, conducted by Lacey, Scott and Siatkowski and Moriarty et al.3,6,7

Vascular malformations of the extraocular muscles, in the absence of haematological abnormalities, can lead to spontaneous intramuscular haemorrhage. 8 The authors saw this phenomenon in one of the studied patients. Moreover, in the current study, the clinical features of the primary malignant non-Hodgkin's lymphoma that involved the left inferior oblique muscle were consistent with the findings of Hornblass et al. ${ }^{9}$

The rhabdomyosarcoma arises from the primitive pluripotential mesenchymal tissue that is present in the soft tissue of the orbit. They may very rarely arise from the extraocular muscle as well. ${ }^{10}$ Porterfield and Zimmerman studied 55 cases of orbital rhabdomyosarcomas and came across just three cases arising from the extraocular muscles. ${ }^{11}$ The current study also reports a case of embryonal rhabdomyosarcoma, arising from the right inferior rectus muscle, in a 5-year boy, who died within two years of diagnosis, due to the recurrent and aggressive nature of the tumour.

The alveolar soft-part sarcoma (ASPS) constitutes $1 \%$ of all the soft tissue sarcomas that affect the young adults. The ASPS has a female preponderance and involves the head and the neck region predominantly. Orbital involvement of ASPS is very rare.12 Abrahams et al. reported a case of orbital ASPS, which replaced the complete insertion of the inferior rectus muscle. 13 Khan and Burke also reported a case of orbital ASPS, which was indistinguishable from the medial rectus muscle, and was surgically excised. ${ }^{14}$ The authors in this study also report a case of alveolar soft-part sarcoma for the first time, which involved the left lateral rectus muscle primarily (Figure 3). The clinical, histopathological and the immunohistochemistry features of the reported ASPS were identical to those already published. ${ }^{12}$

The most common infective process that affects the extraocular muscles is the contiguous involvement of an extraocular muscle from the bacterial sinusitis. ${ }^{3}$ However, this was not seen in this case series. The function of an extraocular muscle can be affected by various pathological processes that result in their enlargement or shrinkage. ${ }^{3}$ The primary extraocular muscle tumours can produce a mass effect, resulting in the displacement of the globe and/or other structures, as well as compromise their function, ${ }^{3}$ as seen in this case series.

At times, the diagnostic dilemmas arise for clinicians, while dealing with swellings associated with chronic progressive muscular dysfunctions. Besides, the primary extraocular muscle tumours do not see a reduction in size following the steroid therapy. Therefore, in these cases, a biopsy is mandatory to rule out malignancy. The primary tumours of the extraocular muscles that have a widespread involvement may respond well to the radiotherapy sessions in divided doses, chemotherapy or palliative debulking.3,15

This is the largest case series on biopsy-proven primary EOM tumours. Besides, the patients of the current study are under rigorous follow-up to address the morbidity associated with the disease/treatment, as well as for rehabilitation, to improve their quality of life.

\section{CONCLUSION}

The primary EOM tumours had an institutional prevalence of $2.96 \%$, classified into five broad categories. All the patients with primary EOM tumours presented with proptosis and impaired ocular motility; whereas, II the primary EOM tumours involved both the recti and the obliques, and were removed surgically with favourable outcomes in most cases.

\section{REFERENCES}

1. Prakash $P$, Nayak BK, Menon V. Abnormal insertion of inferior oblique. Indian J Ophthalmol 1983; 31:21-2.

2. Qi Y, Yu G, Wu Q, Cao WH, Fan YW. Accessory extraocular muscle - a case report and review. Zhonghua Yan KeZaZhi 2011; 47:1111-6.

3. Lacey B, Chang W, Rootman J. Nonthyroid causes of extraocular muscle disease. Surv Ophthalmol 1999; 44:187-213.

4. Hansman ML, Peyster RG, Heiman-Patterson T, Greenfield VS. CT demonstration of extraocular muscle atrophy. J Comput Assist Tomogr 1988; 12:49-51.

5. Mannor GE, Rose GE, Moseley IF, Wright JE. Outcome of orbital myositis. Clinical features associated with recurrence. Ophthalmology 1997; 104:409-13. 
6. Scott IU, Siatkowski RM. Idiopathic orbital myositis. Curr Opin Rheumatol 1997; 9:504-12.

7. Moriarty P, Garner A, Wright JE. Case report of granular cell myoblastoma arising within the medial rectus muscle. $\mathrm{Br} \mathrm{J}$ Ophthalmol 1983; 67:17-22.

8. Hakin KN, McNab AA, Sullivan TJ. Spontaneous haemorrhage within the rectus muscle. Ophthalmology 1994; 101:1631-4.

9. Hornblass A, Jakobiec FA, Reifler DM, Mines J. Orbital lymphoid tumours located predominantly within extraocular muscles. Ophthalmology 1987; 94:688-97.

10. Knowles DM, Jakobiec FA, Potter GD, Jones IS. Ophthalmic striated muscle neoplasms. Surv Ophthalmol 1976; 21: 219-61.
11. Porterfield JF, Zimmerman LE. Rhabdomyosarcoma of the orbit: A clinicopathologic study of 55 cases. Virchows Arch Pathol Anat Physiol Klin Med 1962; 335:329-44.

12. Alkatan $\mathrm{H}, \mathrm{Al}-$ Shedoukhy AA, Chaudhry IA, Al-Ayoubi A. Orbital alveolar soft part sarcoma: Histopathologic report of two cases. Saudi J Ophthalmol 2010; 24:57-61.

13. Abrahams IW, Fenton RH, Vidone R. Alveolar soft-part sarcoma of the orbit. Arch Ophthalmol 1968; 79:185-8.

14. Khan AO, Burke MJ. Alveolar soft-part sarcoma of the orbit. J Pediatr Ophthalmol Strabismus 2004; 41:245-6.

15. Eade EL, Hardy TG, McKelvie PA, McNab AA. Review of extraocular muscle biopsies and utility of biopsy in extraocular muscle enlargement. Br J Ophthalmol 2018; 102:1586-90. 